\title{
Tarsorrafia: aplicações em um Serviço de Córnea
}

\author{
Tarsorrhaphy:applications in a Cornea Service
}

\author{
Patrick Frensel de Moraes Tzelikis ${ }^{1}$ \\ Cristiano Menezes Diniz ${ }^{2}$ \\ Marco Antônio Guarino Tanure ${ }^{3}$ \\ Fernando Cançado Trindade ${ }^{4}$
}

Trabalho realizado no Serviço de Doenças Externas, Catarata e Refrativa do Hospital São Geraldo - Universidade Federal de Minas Gerais.

${ }^{1}$ Ex-Fellow do Serviço de Córnea, Catarata e Doenças Externas do Hospital São Geraldo da Universidade Federal de Minas Gerais.

${ }^{2}$ Médico Assistente do Serviço de Plástica Ocular do Hospital São Geraldo - Universidade Federal de Minas Gerais.

${ }^{3}$ Médico Assistente do Serviço de Córnea e Catarata do Hospital São Geraldo - Universidade Federal de Minas Gerais. Doutor em Oftalmologia pela Faculdade de Medicina da Universidade Federal de Minas Gerais.

${ }^{4}$ Professor Adjunto e Chefe do Serviço de Córnea e Catarata do Hospital São Geraldo - Universidade Federal de Minas Gerais. Doutor em Oftalmologia pela Faculdade de Medicina da Universidade Federal de Minas Gerais

Endereço para correspondência: SQN 203, bloco G, apto. 405 - Brasília (DF) CEP 70833-070

E-mail: tzelikis@zaz.com.br

Recebido para publicação em 12.05.2003

Versão revisada recebida em 07.10.2004

Aprovação em 30.08.2004

Nota Editorial: Por sua análise deste trabalho e por sua anuência na divulgação desta nota, agradecemos ao Dr. Walton Nosé.

\begin{tabular}{|l|}
\hline RESUMO \\
\hline Objetivo: Avaliar as principais indicações de tarsorrafia em Serviço de \\
Córnea, bem como as taxas de sucesso e possíveis complicações do \\
procedimento. Métodos: Foram avaliados, retrospectivamente, todos os \\
pacientes submetidos a tarsorrafia no período de 1 de janeiro de 2002 a 30 \\
de dezembro de 2002 no Hospital São Geraldo, Universidade Federal de \\
Minas Gerais. Avaliou-se idade e sexo do paciente,indicação da tarsorrafia, \\
duração dos sinais e sintomas antes da tarsorrafia, tempopara reepitelização \\
corneana, tipo de tarsorrafia, complicações da tarsorrafia, número de \\
tarsorrafias e duração do acompanhamento. Resultados: Foram encontra- \\
dos 18 pacientes submetidos a tarsorrafia. As indicações para tarsorrafia \\
foram úlcera de exposição (27,8\%), defeito epitelial persistente associado \\
a transplante de córnea (38,8\%), úlcera neurotrófica (11,1\%), síndrome do \\
olho seco (5,6\%), síndrome de Stevens-Johnson (11,1\%) e queimadura \\
química (5,6\%). Dos 18 pacientes que apresentavam defeito epitelial, 15 \\
pacientes (83,3\%) apresentaram epitelização completa com a tarsorrafia. A \\
duração média de sinais e sintomas antes da tarsorrafia foi de $98,7 \pm 48,6$ \\
dias, e o tempo para epitelização após a cirurgia foi de $53,2 \pm 22,8$ dias. Do \\
total de 18 tarsorrafias, duas (11,1\%) foram temporárias e 16 ( $88,9 \%)$ \\
permanentes. As complicações relacionadas ao procedimento cirúrgico \\
foram abertura precoce da tarsorrafia, triquíase e granuloma piogênico. \\
Conclusão: A tarsorrafia se mostrou procedimento de fácil realização, \\
sendo bastante segura e eficaz no tratamento de defeitos epiteliais, com \\
taxa de sucesso de $83,3 \%$ e com poucas complicações. \\
\hline
\end{tabular}

Descritores: Pálpebras/cirurgia; Procedimentos cirúrgicos oftálmicos; Úlcera da córnea; Ceratoplastia penetrante; Epitélio da córnea/cirurgia; Prestação de cuidados de saúde

\section{INTRODUÇ̃̃̃O}

A tarsorrafia é um procedimento cirúrgico que consiste na fusão da pálpebra superior com a inferior ${ }^{(1)}$. Trata-se de uma técnica simples que pode ser realizada isoladamente ou associada a outras cirurgias oculoplásticas para tratamento de uma série de doenças, como lagoftalmo, paralisia facial, ectrópio, entrópio, proptose, doença tireoidiana entre outras ${ }^{(2-3)}$. A tarsorrafia é comumente utilizada em Serviços de Córnea e Doenças Externas como uma opção de tratamento para se obter uma proteção da córnea em casos de proptose ou para acelerar a cicatrização de úlceras corneanas ocasionadas, por exemplo, por paralisia facial. É um procedimento extremamente útil para tratamento de defeitos epiteliais persistentes secundários a ceratopatia de exposição, ceratopatia neurotrófica e na síndrome do olho seco. Pode ser associado a outras cirurgias como ceratoplastia penetrante $(\mathrm{CPP})^{(4)}$.

O objetivo deste trabalho é avaliar as indicações, eficácia e complicações relacionadas à tarsorrafia em um Serviço de Córnea e Doenças Externas. 


\section{MÉTODOS}

Foram avaliados retrospectivamente todos os pacientes submetidos à tarsorrafia no período de $1^{\circ}$ de janeiro de 2002 a 30 de dezembro de 2002, no Serviço de Córnea, Catarata e Doenças Externas do Hospital São Geraldo - Universidade Federal de Minas Gerais. Foram incluídos neste estudo pacientes com período mínimo de acompanhamento de 2 meses pós tarsorrafia.

As informações obtidas para este trabalho foram idade e sexo do paciente, indicação da cirurgia, duração dos sinais e sintomas antes do procedimento, tempo necessário para reepitelização após a cirurgia, tipo de tarsorrafia (temporária ou permanente), complicações, número de tarsorrafias, e duração do acompanhamento.

Todos os pacientes foram submetidos inicialmente a tratamento clínico com lubrificante e pomada de antibiótico. Alguns fizeram uso de lente de contato terapêutica (LCT), como, por exemplo, os pacientes com úlcera neurotrófica por herpes, com cobertura antibiótica devido a LCT. Apenas um paciente, portador de olho seco grave, teve seu ponto lacrimal ocluído. A tarsorrafia era indicada nos casos onde não se obtinha sucesso na tentativa de reepitelização com os procedimentos descritos anteriormente. Ao se diagnosticar clinicamente uma doença de curta evolução, a tarsorrafia realizada era temporária e, quando se tratava de uma doença de longa duração ou irreversível, o procedimento era realizado de forma definitiva. As tarsorrafias foram feitas pelo próprio cirurgião do Serviço de Córnea e Doenças Externas imediatamente após a realização de procedimentos tais como a ceratoplastia penetrante. Nos casos onde era necessário apenas o procedimento para diminuir a abertura palpebral, o mesmo era realizado pelo cirurgião do Serviço de Plástica Ocular.

A tarsorrafia temporária foi realizada com 1 ou 2 pontos em "U" com nylon 5-0 passados sobre protetores de silicone ou esponja. Passava-se a agulha de cima para baixo, a $4 \mathrm{~mm}$ da margem superior saindo exatamente na linha cinzenta, e penetrando a seguir, na pálpebra inferior pela mesma linha cinzenta. Os pontos eram passados em "U" e apertados externamente sobre esponjas protetoras ${ }^{(1)}$. Nas tarsorrafias permanentes, a lamela anterior e posterior eram separadas na linha cinzenta com um bisturi no 11 . A borda conjuntival da margem palpebral posterior era removida com tesoura. Passava-se uma sutura contínua com categute 6-0 unindo as lamelas posteriores das duas pálpebras, uma segunda sutura em "U" unindo as lamelas anteriores das pálpebras superior e inferior com nylon 5-0 ancorados em pedaços de esponja ou silicone. Em alguns casos realizava-se apenas a sutura das lamelas anteriores ancoradas nos protetores ${ }^{(5)}$.

Os dados dos pacientes foram armazenados e analisados no programa EPI-INFO, versão 6.04 - CDC, Atlanta.

\section{RESULTADOS}

Foram avaliados retrospectivamente 18 pacientes submetidos à tarsorrafia. Seis pacientes $(33,3 \%)$ eram do sexo masculino e $12(66,7 \%)$ do sexo feminino. A idade dos pacientes variou de 17 a 85 anos com média de 49,8 $\pm 19,2$ anos.

As indicações de tarsorrafia foram defeitos epiteliais persistentes associados à ceratoplastia penetrante $(\mathrm{CPP})$ em sete $(38,8 \%)$ casos, úlcera de exposição em cinco $(27,8 \%)$, úlcera neurotrófica em dois $(11,1 \%)$, síndrome de Stevens-Johnson em dois $(11,1 \%)$, queimadura química em um $(5,6 \%)$, e síndrome do olho seco em um $(5,6 \%)$ (Tabela 1 e 2 ). A doença base responsável pelos sete casos de CPP foi: úlcera infecciosa em três $(42,8 \%)$, úlcera de Mooren em um $(14,3 \%)$, glaucoma congênito em um (14,3\%), ceratopatia bolhosa em um (14,3\%), e esclerodermia em um (14,3\%). A tarsorrafia foi realizada no mesmo ato operatório da CPP em seis casos, numa tentativa de antecipar o defeito de reepitelização, muito comum em olhos com comprometimento límbico extenso e olhos que já apresentavam defeito epitelial previamente.

De um total de 18 olhos, três (16,7\%) pacientes não obtiveram melhora do defeito epitelial com a tarsorrafia. Um apresentava esclerodermia, e os outros dois, queimadura química e defeito epitelial persistente após ceratoplastia penetrante. No caso de esclerodermia, foi realizada tarsorrafia após a 1a $\mathrm{CPP}$ e mesmo assim o quadro evoluiu com "melting" corneano sendo então procedida nova CPP juntamente com tarsorrafia. Entretanto, houve persistência do defeito epitelial com necessidade de se realizar recobrimento conjuntival. O caso ainda foi agravado por uma ceratoconjuntivite sicca grave e importante fibrose do fórnice conjuntival com lagoftalmo. Atualmente, a paciente se encontra em acompanhamento sob imunossupressão (Serviço de Reumatologia do HC - UFMG) e mantendo quadro estável. Os dois outros pacientes foram submetidos a recobrimento conjuntival e a evisceração, respectivamente (Tabela 2).

Do total de 18 tarsorrafias, $16(88,9 \%)$ foram permanentes e duas $(11,1 \%)$ temporárias (dois pacientes com úlcera de exposição por paralisia facial). Das 16 tarsorrafias permanentes, seis $(37,5 \%)$ ainda não foram retiradas após acompanhamento de $288 \pm 136$ dias. A duração da tarsorrafia temporária nos dois casos foi de 4 semanas.

Em relação ao local das tarsorrafias, nove $(50,0 \%)$ foram realizadas centralmente, quatro $(22,2 \%)$ temporalmente e cinco $(27,8 \%)$ tanto nasal como temporalmente.

A duração dos sinais (hiperemia conjuntival, erosões corneanas e úlceras) e sintomas (sensação de corpo estranho, ardência, fotofobia, queimação), antes da tarsorrafia foi de $98,7 \pm 48,6$ dias, variando de 34 a 210 dias, e após a tarsorrafia de $53,2 \pm 22,8$ dias, variando de 19 a 90 dias. Foi realizada análise de variância com uma comparação das médias entre a duração dos sinais e sintomas antes e após a tarsorrafia usando o método de Satterhwaite com valor de $\mathrm{p}=0,0858$. Apesar de se observar uma melhora rápida com o uso da 


\begin{tabular}{|c|c|c|c|c|c|c|}
\hline Caso & Indicação & Sexo & $\begin{array}{l}\text { Idade } \\
\text { (anos) }\end{array}$ & $\begin{array}{l}\text { Duração dos sinais } \\
\text { e sintomas (dias) }\end{array}$ & $\begin{array}{c}\text { Tempo para } \\
\text { reepitelização (dias) }\end{array}$ & $\begin{array}{c}\text { Acompanhamento } \\
\text { (dias) }\end{array}$ \\
\hline 1 & Úlcera neurotrófica por Herpes zoster & $\mathrm{F}$ & 64 & 98 & 37 & 172 \\
\hline 2 & Defeito epitelial pós-CPP & M & 68 & 53 & 72 & 395 \\
\hline 3 & Úlcera exposição por paralisia facial & $\mathrm{F}$ & 67 & 92 & 37 & 408 \\
\hline 4 & Úlcera exposição por paralisia facial & $\mathrm{F}$ & 34 & 92 & 90 & 114 \\
\hline 5 & Olho seco & $\mathrm{F}$ & 40 & 210 & 19 & 309 \\
\hline 6 & Síndrome de Stevens - Johnson & $\mathrm{F}$ & 55 & 90 & 56 & 236 \\
\hline 7 & Úlcera exposição por trauma & M & 43 & 63 & 87 & 358 \\
\hline 8 & Úlcera neurotrófica por Herpes simplex & $\mathrm{F}$ & 52 & 177 & 64 & 120 \\
\hline 9 & Úlcera exposição por miopatia & M & 28 & 120 & 19 & 69 \\
\hline 10 & Defeito epitelial pós-CPP & M & 85 & 57 & 37 & 162 \\
\hline 11 & Síndrome de Stevens - Johnson & $\mathrm{F}$ & 30 & 45 & 27 & 210 \\
\hline 12 & Úlcera exposição por paralisia facial & $\mathrm{F}$ & 48 & 94 & 60 & 425 \\
\hline 13 & Defeito epitelial pós-CPP & $\mathrm{F}$ & 27 & 34 & 59 & 227 \\
\hline 14 & Defeito epitelial pós-CPP & M & 17 & 131 & 61 & 291 \\
\hline 15 & Defeito epitelial pós-CPP & M & 69 & 125 & 73 & 348 \\
\hline
\end{tabular}

\begin{tabular}{|c|c|c|c|c|}
\hline Caso/idade/sexo & Diagnóstico & Cirurgia prévia & Doenca sistêmica & Evolucão \\
\hline $1 / 34 / F$ & $\begin{array}{l}\text { Defeito epitelial } \\
\text { persistente após CPP }\end{array}$ & CPP & Esclerodermia & $\begin{array}{l}1^{\circ} \text { tarsorrafia, } 20 \mathrm{CPP}+\text { tarsorrafia, } \\
\text { depois submetida a recobrimento } \\
\text { conjuntival e nova tarsorrafia }\end{array}$ \\
\hline $2 / 65 / F$ & Queimadura química & - & DM & $\begin{array}{l}\text { 1 Tarsorrafia, 2ํ CPP + tarsorrafia, } \\
\text { 3 evisceração }\end{array}$ \\
\hline $3 / 72 / F$ & $\begin{array}{l}\text { Defeito epitelial } \\
\text { persistente após CPP }\end{array}$ & $\mathrm{FEC}+\mathrm{LIO}$ & $\mathrm{DM}$ & $\begin{array}{l}1^{\circ} \text { tarsorrafia, } 2^{\circ} \mathrm{CPP}+\text { tarsorrafia, } 3^{\circ} \mathrm{CPP}+ \\
\text { tarsorrafia, } 4^{\circ} \text { recobrimento conjuntival }\end{array}$ \\
\hline
\end{tabular}

tarsorrafia, não foi verificado, portanto, nenhuma diferença estatisticamente significante $(\mathrm{p}>0,05)$, talvez por se tratar de uma amostra pequena.

Dentre as complicações relacionadas a este procedimento cirúrgico, dois $(11,1 \%)$ pacientes apresentaram abertura precoce da tarsorrafia, três $(16,7 \%)$ desenvolveram triquíase e um $(5,6 \%)$ granuloma piogênico. A maioria das complicações ocorreu na tarsorrafia permanente com exceção de uma abertura precoce em paciente com tarsorrafia temporária.

\section{DISCUSSÃO}

Tarsorrafia é um procedimento que objetiva o fechamento das pálpebras, de forma temporária ou definitiva. Existem hoje basicamente quatro tipos diferentes de tarsorrafia que se encontram à disposição do cirurgião, que deve escolher a técnica mais adequada para determinada situação. O primeiro tipo é a tarsorrafia temporária. Como já descrito, pode ser usada em situações que indicam uma doença com evolução curta. Geralmente as suturas permanecem por 4 a 6 semanas $^{(6)}$.

O segundo tipo, a tarsorrafia permanente, é realizado atra- vés da incisão da linha cinzenta possibilitando uma maior adesão entre as margens palpebrais opostas. A técnica do retalho tarsoconjuntival na qual se desliza uma pálpebra sobre a outra, após a retirada de um segmento tarsoconjuntival triangular é, também, considerada um tipo de tarsorrafia permanente ${ }^{(1,7)}$.

O terceiro tipo de tarsorrafia consiste na mobilização de retalhos tarsais opostos. Inicialmente, divide-se a pálpebra superior e inferior em lamela anterior e posterior. Posteriormente, retira-se a lamela anterior da pálpebra superior e a lamela posterior da pálpebra inferior ou vice-versa e unem-se as lamelas opostas promovendo-se um encaixe adequado ${ }^{(5,7)}$.

O quarto tipo de tarsorrafia é aquele no qual não se faz uso de suturas. As margens palpebrais são unidas através de cola $^{(8-9)}$, fitas adesivas, ou através da aplicação de toxina botulínica para paralisar o músculo levantador da pálpebra ${ }^{(10)}$.

Pacientes que apresentam olhos normais quando desenvolvem abrasões corneanas de tamanho pequeno a moderado geralmente obtém uma reepitelização num prazo de 12 a 24 horas. No caso de abrasões corneanas extensas, espera-se um tempo maior para que ocorra a reepitelização, freqüentemente entre uma a duas semanas ${ }^{(11)}$. Reserva-se o termo defeito epitelial persistente quando não se obtém a reepitelização corneana 
num prazo esperado para que ela aconteça. Várias formas de tratamento são descritas na literatura, entre elas: pomadas e lubrificantes, soro autólogo, lente de contato, oclusão, membrana amniótica, recobrimento conjuntival e tarsorrafia ${ }^{(12-15)}$.

Trabalhos na literatura mostram taxas de sucesso para reepitelização entre $80 \%$ a $100 \%$ dos casos com uso de tarsorrafia $^{(5-6,8)}$. Neste trabalho, foi obtida uma taxa de sucesso de $83,3 \%$ dos casos sendo que em apenas três $(16,7 \%)$ não ocorreu reepitelização. Nestes três pacientes onde a tarsorrafia não foi eficaz, existiam doenças sistêmicas as quais comprometem o equilíbrio e a dinâmica do processo de cicatrização epitelial. No trabalho publicado em $2001^{(5)}$, os autores obtiveram uma taxa de sucesso de $90,9 \%$ utilizando tarsorrafia em pacientes com defeito epitelial com um tempo de reepitelização da córnea de $18 \pm 2$ dias, sendo que neste estudo o tempo para reepitelização foi de 53,2 $\pm 22,8$ dias. Neste mesmo trabalho, as principais indicações de tarsorrafia foram defeitos epiteliais associados com úlcera neurotrófica $(32,5 \%)$, transplante de córnea $(28,6 \%)$, pós-infecção $(14,3 \%)$ e ceratopatia de exposição $(10,4 \%)^{(5)}$. No presente trabalho, a principal razão foi defeito epitelial associado com transplante de córnea (38,8\%), seguido por ceratopatia de exposição $(27,8 \%)$, e úlcera neurotrófica $(11,1 \%)$. As indicações foram basicamente as mesmas, porém com distribuição percentual diferente.

Neste estudo, o período para reepitelização variou conforme a gravidade das doenças tratadas, sendo que as patologias que apresentavam maior comprometimento límbico, como, por exemplo, as pós-ceratoplastia penetrante e paralisia facial com pouca ou nenhuma função do músculo orbicular, combinada com hipoestesia corneana e deficiência do reflexo de Bell, foram as que apresentaram taxas de reepitelização mais lenta.

A dinâmica fisiológica da superfície ocular e do filme lacrimal ainda permanece sem algumas respostas. Vários fatores parecem estar relacionados a uma reepitelização deficiente em alguns olhos. Fatores neurotróficos originados do nervo trigêmeo, bem como o filme lacrimal e o próprio ato de pestanejar estão interligados e conferem à superfície ocular um equilíbrio no processo de regeneração e cicatrização epitelial ${ }^{(16)}$. Olhos com perda de estímulos tróficos, como, por exemplo, Herpes zoster, com aumento da exposição da superfície ocular e deficiência de filme lacrimal apresentam maiores riscos para desenvolverem defeitos epiteliais persistentes. As úlceras neurotróficas presente nos pacientes 1 e 8 (Tabela 1), causadas respectivamente por infecção por Herpes zoster e Herpes simplex, resultaram em um comprometimento da sensibilidade corneana associada a uma diminuição na produção de lágrima. Nesses casos, observa-se inicialmente uma irregularidade epitelial e uma epiteliopatia punctata leve a qual, com o tempo, pode progredir para um defeito epitelial maior. Geralmente, o defeito epitelial em úlceras neurotróficas é oval e com bordas regulares e lisas. Estes defeitos, se não tratados de forma adequada, podem evoluir para ulceração estromal, necrose, perfuração, e infecção secundária ${ }^{(17)}$.

Dentre as complicações precoces mais freqüentes em pósoperatórios de CPP, encontram-se os defeitos epiteliais persis- tentes. Se a superfície ocular do paciente apresenta-se saudável, a reepitelização geralmente ocorre de forma rápida e sem intercorrências. No entanto, existem situações que, mesmo sem nenhuma doença na superfície ocular, defeitos epiteliais podem ocorrer. Pacientes com ceratoconjuntivite sicca, queimadura por álcali, ceratopatia neurotrófica ou de exposição podem dificultar ainda mais o processo de reepitelização corneana e gerar defeitos epiteliais persistentes no pós-operatório de uma CPP. Neste trabalho, 6 pacientes apresentaram defeito epitelial no pós-operatório de CPP. Em pacientes onde se espera problemas na fase de reepitelização corneana, o uso de uma córnea doadora com um bom epitélio e um curto período de preservação é essencial para minimizar o problema de reepitelização. Durante o ato operatório, deve-se ter cuidado no manuseio e manter o epitélio corneano sempre lubrificado. Uma boa coaptação entre a junção doador-receptor é fundamental para a migração epitelial. Em situações nas quais mesmo com todos os cuidados se desenvolve um defeito epitelial, a tarsorrafia se torna uma excelente opção terapêutica.

As úlceras de exposição, presentes em 5 pacientes no presente estudo, são causa importante de defeito epitelial. Por um motivo neurológico ou mecânico, observa-se um aumento da fenda palpebral com um inadequado fechamento ocular levando a uma instabilidade do filme lacrimal e olho seco. Além disso, nas úlceras por paralisia de Bell (paralisia facial periférica) observou-se uma redução ou ausência da sensibilidade corneana em $10 \%$ dos pacientes, presumidamente por um mecanismo de adaptação ${ }^{(18)}$.

A tarsorrafia teria a vantagem de diminuir a taxa de evaporação da lágrima por reduzir a abertura palpebral. Além disso, a restrição do pestanejar com a tarsorrafia causaria uma diminuição do efeito traumático que o movimento palpebral confere à superfície ocular em recuperação. O local ideal para a tarsorrafia ainda é motivo de controvérsia. No Hospital São Geraldo - HC/ UFMG, geralmente utiliza-se apenas uma tarsorrafia, central ou temporal, geralmente tentando situar a tarsorrafia no local correspondente ao defeito epitelial. Para se obter uma oclusão extensa, realiza-se uma nasal e outra temporal. Não existem trabalhos na literatura comparando as diferentes localizações da tarsorrafia e seu resultado na reepitelização corneana.

Outras formas de tratamento para defeitos epiteliais também apresentam excelentes resultados na literatura. Em 2001 num trabalho realizado em Boston - EUA, demonstraram uma reepitelização corneana completa com uso de membrana amniótica (MA) em 70\% dos pacientes com defeito epitelial por diferentes patologias num período médio de 25,5 dias ${ }^{(17)}$. Em outro estudo realizado em Singapura em 2001, os autores obtiveram uma reepitelização corneana completa em $83 \%$ dos pacientes com uso de LCT num período médio de 1,1 mês ${ }^{(18)}$. No presente trabalho, apenas os pacientes com úlcera neurotrófica foram tratados com LCT quando não responderam ao tratamento inicial com pomada lubrificante, porém não alcançaram uma reepitelização corneana completa, sendo então indicada a tarsorrafia. 
Os olhos, com redução da abertura palpebral, como ocorre nas tarsorrafias, permitem que o epitélio corneano receba uma maior quantidade de oxigênio quando comparado à oclusão completa do olho. A abertura da pálpebra possibilita ainda o emprego adjuvante de medicação tópica e o exame da córnea através da movimentação ocular. A partir destas observações a tarsorrafia mostra-se como uma forma de tratamento mais vantajosa quando comparada com a oclusão ocular. A oclusão completa ou por meio de lentes de contato causa uma redução da distribuição do filme lacrimal, diminui a eliminação de partículas, de células mortas e aumenta a temperatura local favorecendo o crescimento bacteriano. Estudos que compararam a tarsorrafia com a oclusão compressiva ou com as lentes de contato foram favoráveis a tarsorrafia ${ }^{(12,19-20)}$.

Diversas complicações já foram descritas com o uso de tarsorrafia, entre elas: triquíase ${ }^{(5)}$, deformidades da margem palpebral $^{(21)}$, granulomas de sutura ${ }^{(5)}$, e abertura prematura da tarsorrafia ${ }^{(22)}$. No presente trabalho a principal complicação foi a triquíase que ocorreu em três $(16,7 \%)$ pacientes, e que foi tratada com sucesso através da aplicação de laser de argônio na base do cílio. Em alguns trabalhos na literatura observa-se certa relutância por parte dos pacientes para realização da tarsorrafia por motivos estéticos ${ }^{(5,22)}$. Nenhum paciente se mostrou contrário ao procedimento no nosso serviço.

\section{CONCLUSÃO}

A tarsorrafia, seja ela temporária ou permanente, mostrou ser um procedimento bastante seguro e eficaz para o tratamento de defeitos epiteliais persistentes, alcançando uma taxa de sucesso de $83,3 \%$, com poucas complicações.

\section{ABSTRACT}

Purpose: To evaluate the main indications for tarsorrhaphy in a Corneal Service, as well as success rate and possible complications. Methods: All patients who underwent tarsorrhaphy from January $1^{\text {st }}, 2002$ to December $30^{\text {th }}, 2002$, in São Geraldo Eye Hospital - Federal University of Minas Gerais were evaluated retrospectively. Reviewed data included patient's sex and age, indication for tarsorrhaphy, duration of signs and symptoms before tarsorrhaphy, time for epithelial healing after tarsorrhaphy, type of tarsorrhaphy, complications, follow-up time. Results: Eighteen patients underwent tarsorrhaphy. The indications for a tarsorrhaphy were exposure keratopathy (27.8\%), persistent epithelial defect associated with penetrating keratoplasty $(38.8 \%)$, neurotrophic ulcer $(11.1 \%)$, dry eye syndrome (5.6\%), Stevens-Johnson syndrome (11.1\%), and chemical burn $(5.6 \%)$. The epithelial defect resolved completely in 15 patients (83.3\%). Mean duration of signs and symptoms before tarsorrhaphy was $98.7 \pm 48.6$ days, and time to heal after tarsorrhaphy was $53.2 \pm 22.8$ days. Of the 18 procedures, two $(11.1 \%)$ were temporary and $16(88.9 \%)$ permanent. Complications after tarsorrhaphy were premature opening of the tarsorrhaphy, trichiasis and pyogenic granuloma. Conclusion: Tarsorrhaphy is a simple procedure, being also very safe and effective in the management of nonhealing epithelial defects, with a success rate of $83.3 \%$ and with minor complications.

Keywords: Eyelids /surgery; Ophthalmologic surgical procedures; Corneal ulcer; Keratoplasty, penetrating; Epithelium, Corneal/surgery; Delivery of health care

\section{REFERÊNCIAS}

1. Moura EM da. Blefarorrafia e tarsorrafia. In: Belfort Jr R, Kara-José N. Córnea clínica: cirúrgica. São Paulo: Roca; 1996. p.457-62.

2. American Academy of Ophthalmology. Basic and Clinical Science Course. External Disease and Cornea. Section 8. San Francisco: American Academy of Ophthalmology; 2001. p.392-4.

3. Stewart WB, editor. Surgery of the eyelid, orbit and lacrimal system. In: Stewart WB. Plastic surgery. San Francisco: American Academy of Ophthalmology; 1994. (Ophthalmology monographs, 8).

4. Verdier DD. Penetrating keratoplasty. In: Kracher JH, Mannis MJ, Holland EJ, editors. Cornea. St Louis: Mosby; 1997. v.3, p.1581-92.

5. Cosar CB, Cohen EJ, Rapuano CJ, Maus M, Penne RP, Flanagan JC, et al. Tarsorrhaphy: clinical experience from a cornea practice. Cornea. 2001;20(8): 787-91.

6. Rapoza PA, Harrison DA, Bussa JJ, Prestowitz WF, Dortzbach RK. Temporary sutured tube-tarsorrhaphy: reversible eyelid closure technique. Ophthalmic Surg. 1993;24(5):328-30.

7. Stamler JF, Tse DT. A simple and reliable technique for permanent lateral tarsorrhaphy. Arch Ophthalmol. 1990;108(1):125-7.

8. Donnenfeld ED, Perry HD, Nelson DB. Cyanoacrylate temporary tarsorrhaphy in the management of corneal epithelial defects. Ophthalmic Surg. 1991;22 (10):591-3.

9. Bonatti JA, Cresta FB. Cola de fibrina e cianoacrilato. In: Alves MR, KaraJosé N. Conjuntiva cirúrgica. São Paulo: Roca; 1999. p.221.

10. Kirkness CM, Adams GC, Dilly PN, Lee JP. Botulinum toxin A - induced protective ptosis in corneal disease. Ophthalmology. 1988;95(4):437-80.

11. Hamill MB. Corneal injury. In: Krachmer JH, Mannis MJ, Holland EJ, editors. Cornea. St Louis: Mosby; 1997. v.2, p.1403-22.

12. Baum J. The advantages of partial patching or tarsorrhaphy over complete eyelid closure. Am J Ophthalmol. 1987;103(3Pt1):339-40.

13. Moreira SMB, Moreira H. Lentes de contato terapêuticas. In: Moreira SMB, Moreira H. Lentes de contato. Rio de Janeiro: Cultura Médica; 1993. p.259.

14. Arieta CEL. Recobrimento conjuntival. In: Alves MR, Kara-José N. Conjuntiva cirúrgica. São Paulo: Roca; 1999. p.231.

15. Shimazaki J, Yang HY, Tsubota K. Amniotic membrane transplantation for ocular surface reconstruction in patients with chemical and thermal burns. Ophthalmology. 1997;104(12):2068-76.

16. Dua HS, Gomes JA, Singh A. Corneal epithelial wound healing. Br J Ophthalmol. 1994;78(5):401-8.

17. Letko E, Stechschulte SU, Kenyon KR, Sadeq N, Romero TR, Samson CM, et al. Amniotic membrane inlay and overlay grafting for corneal epithelial defects and stromal ulcers. Arch Ophthalmol. 2001;119(5):659-63.

18. Lim L, Tan DT, Chan WK. Therapeutic use of Bausch \& Lomb PureVision contact lenses. CLAO J. 2001;27(4):179-85.

19. Wagoner MD, Steinert R. Temporary tarsorrhaphy enhances reepithelialization after epikeratoplasty. Arch Ophthalmol. 1988;106(1):13-4.

20. Panda A, Pushker N, Bageshwar LM. Lateral tarsorrhaphy: is it preferable to patching? Cornea. 1999;18(3):299-301. Commented in: Cornea. 1999;18(6): 739-40.

21. Perusse P. Localized distichiasis after tarsorrhaphy. Am J Ophthalmol. 1992; 114(1):104-5.

22. Gossman MD, Bowe BE, Tanenbaum M. Reversible suture tarsorrhaphy for eyelid malposition and keratoplasty. Ophthalmic Surg. 1991;22(4):237-9. 